\title{
Linear Processing with Pregroups
}

\author{
Anne Preller \\ LIRMM-CNRS \\ 161, rue Ada \\ Montpellier, France \\ preller@lirmm.fr *
}

\begin{abstract}
Pregroup grammars have a cubic recognition algorithm. Here, we define a correct and complete recognition and parsing algorithm and give sufficient conditions for the algorithm to run in linear time. These conditions are satisfied by a large class of pregroup grammars, including grammars that handle coordinate structures and distant constituents.
\end{abstract}

\section{Introduction}

Pregroup grammars were introduced in [Lambek 99] as an algebraic tool for the syntactical analysis of sentences and shown to be weakly equivalent to context free grammars by [Buszkowski]. Therefore the usual cubic recognition algorithms of [CYK] or [Earley] for context-free grammars can be used for pregroup grammars after transforming them into rewrite-grammars. Cubic algorithms tailored to pregroups were given in 2004 independently by [Oehrle] and [Degeilh-Preller]. The algorithm proposed in the latter improves on the usual algorithms for context-free languages by the fact the constant factor of $n^{3}$ no longer depends on the number of rules or the number of symbols in the grammar and that it also works for infinite dictionaries. In particular it shows that proof search in compact bilinear logic is proportional to the cube of the length of the string of simple types.

However, cubic complexity easily exceeds human memory capacity and therefore natural languages are most likely to have grammars which permit linear processing. Two observations are important as far as pregroup grammars are concerned: A low number of types per word or a low number of basic types does not diminish the complexity of the recognition algorithm nor the number of different parsings. As an example, consider the rigid dictionary listing three words, each with a single type, say $s a^{\ell}, a a^{\ell}$ and $a a^{r} a$ respectively. The general algorithm still requires cubic time. Moreover, the number of different parsings

\footnotetext{
*The original publication is available at www.springerlink.com
} 
increases exponentially with the length of the string. It seems appropriate to divide the task of finding a linear recognition and parsing algorithm as follows

1. choose a lexical entry for each word, i.e. choose a meaning

2. decide if the choice of types has a reduction to the sentence type and, if there is one, provide at least one such reduction.

We impose conditions on the dictionary concerning both aspects. After the basic notions on pregroups and reductions we present a dictionary covering distant dependencies and coordination in Section 3. as an example of the language fragments for which complete linear parsing is possible. In Section 4. we introduce a parsing algorithm and indicate a class of dictionaries for which it is complete. Restricting the class of dictionaries even more we make the parsing algorithm linear. These conditions are relaxed in Sections 5. where we define the minimal reduction, representative for all reductions to the sentence type. Finally in Section (6), we modify the earlier parsing algorithm by incorporating minimal reductions and define a class of dictionaries for which it is complete.

\section{Geometry of derivations}

We briefly recall the definition of pregroups and the construction of a freely generated pregroup defined in [Lambek 99]. As we are interested in parsing and semantical interpretation, we look at the actual derivations in a free pregroup concentrating on derivations that consist of generalized contractions only. The geometrical structure of these derivations is at the base of the semantical interpretation in Section 3.

A preordered monoid $\langle P, 1, \cdot, \rightarrow\rangle$ is a set with at least one element $1 \in P$, a binary operation $\cdot$ and a binary relation $\rightarrow$ satisfying for all $x, y, z, u, v \in P$

$$
\begin{aligned}
& 1 \cdot x=x=x \cdot 1 \\
& (x \cdot y) \cdot z=x \cdot(y \cdot z) \\
& x \rightarrow x \\
& x \rightarrow y \text { and } y \rightarrow z \text { imply } x \rightarrow z \\
& x \rightarrow y \text { implies } u \cdot x \cdot v \rightarrow u \cdot y \cdot v .
\end{aligned}
$$

The dot denotes multiplication and is generally omitted. The arrow $\rightarrow$ denotes the preorder.

A pregroup is a preordered monoid in which every element $x$ has both a left adjoint $x^{\ell}$ and a right adjoint $x^{r}$ satisfying

$$
\begin{array}{ll}
\text { (Contraction) } & x^{\ell} x \rightarrow 1, x x^{r} \rightarrow 1 \\
\text { (Expansion) } & 1 \rightarrow x^{r} x, 1 \rightarrow x x^{\ell} .
\end{array}
$$

One derives

(1.) $\quad x \rightarrow y$ if and only if $y^{\ell} \rightarrow x^{\ell}$ if and only if $y^{r} \rightarrow x^{r}$,

(2.) $\quad x \rightarrow y$ if and only if $x y^{r} \rightarrow 1$ if and only if $y^{\ell} x \rightarrow 1$.

The free pregroup $P(B)$ generated by a partially ordered set of basic types $B=\{a, b, \ldots\}$ is characterized in [Lambek 99] as the preordered free monoid 
generated from the set of simple types $\Sigma$ consisting of the basic types and their iterated adjoints

$$
\Sigma=\left\{a^{(z)}: a \in B, z \in \mathbb{Z}\right\}
$$

where $a^{(0)}$ is identified with $a$. Note that the empty string is not a simple type. Elements $X \in P(B)$ are called types. They are strings of the form

$$
X=a_{1}^{\left(z_{1}\right)} \ldots a_{k}^{\left(z_{k}\right)}
$$

where $a_{1}, \ldots, a_{k}$ are basic types and $z_{1}, \ldots, z_{k}$ are integers. The unit 1 denotes the empty string and multiplication is the same as concatenation. The left and right adjoints of a type are defined by

$$
\begin{aligned}
& \left(a_{1}^{\left(z_{1}\right)} \ldots a_{k}^{\left(z_{k}\right)}\right)^{\ell}=a_{k}^{\left(z_{k}-1\right)} \ldots a_{1}^{\left(z_{1}-1\right)} \\
& \left(a_{1}^{\left(z_{1}\right)} \ldots a_{k}^{\left(z_{k}\right)}\right)^{r}=a_{k}^{\left(z_{k}+1\right)} \ldots a_{1}^{\left(z_{1}+1\right)} .
\end{aligned}
$$

Hence, identifying $a \in B$ with $a^{(0)} \in \Sigma$ we have

$$
a^{\ell \ell}=a^{(-2)}, a^{\ell}=a^{(-1)}, a=a^{(0)}, a^{r}=a^{(1)}, a^{r r}=a^{(2)} \text { etc. }
$$

If $t=a^{(z)}$ we call $z$ the iterator of $t$.

Finally, the preorder on types is defined as the transitive closure of the union of the following three relations

$$
\begin{array}{ll}
\text { (Induced step) } & X a^{(z)} Y \rightarrow X b^{(z)} Y \\
\text { (Generalized contraction) } & X a^{(z)} b^{(z+1)} Y \rightarrow X Y \\
\text { (Generalized expansion) } & X Y \rightarrow X a^{(z+1)} b^{(z)} Y
\end{array}
$$

where either $z$ is even and $a \rightarrow b$ or $z$ is odd and $b \rightarrow a$. Here $X, Y$ are (possibly empty) strings of simple types and $a$ and $b$ are basic.

As usual, a substring of a string $s_{1} \ldots s_{n}$ has the form $s_{i_{0}} \ldots s_{i_{k}}$ where $1 \leq$ $i_{0}<\ldots<i_{k} \leq n$. A segment $s_{l} \ldots s_{m}$ is a substring such that $l=i_{0}, l+1=i_{1}$, $\ldots, l+k=i_{k}$ and $m=l+k$. By convention, if $m<l$, then $s_{l} \ldots s_{m}$ stands for the empty segment.

The property which makes the theory of pregroups decidable is expressed in the so-called

Switching Lemma (Proposition 2 of [Lambek 99]):

Let $s_{1}, \ldots, s_{n}$ and $t_{1}, \ldots, t_{m}$ be simple types. Then $s_{1} \ldots s_{n} \rightarrow t_{1} \ldots t_{m}$ if and only if there are a substring $s_{i_{1}} \ldots s_{i_{k}}$ of $s_{1} \ldots s_{n}$ and a substring $t_{i_{1}} \ldots t_{i_{k}}$ of $t_{1} \ldots t_{m}$ such that

$$
\begin{gathered}
s_{1} \ldots s_{n} \rightarrow s_{i_{1}} \ldots s_{i_{k}} \rightarrow t_{i_{1}} \ldots t_{i_{k}} \rightarrow t_{1} \ldots t_{m} \\
s_{i_{p}} \rightarrow t_{i_{p}}, \text { for } 1 \leq p \leq k
\end{gathered}
$$

where $s_{i_{1}} \ldots s_{i_{k}}$ is obtained from $s_{1} \ldots s_{n}$ by generalized contractions only, $t_{1} \ldots t_{m}$ is obtained from $t_{i_{1}} \ldots t_{i_{k}}$ by generalized expansions only and $t_{i_{1}} \ldots t_{i_{k}}$ is obtained from $s_{i_{1}} \ldots s_{i_{k}}$ by induced steps only. 
A string of simple types $s_{1} \ldots s_{n}$ is irreducible if no two adjacent simple types satisfy $s_{i} s_{i+1} \rightarrow 1$. In particular, the empty string 1 is irreducible. A type $s_{i_{1}} \ldots s_{i_{k}}$ is an irreducible form of $s_{1} \ldots s_{n}$ if it is irreducible and obtained from $s_{1} \ldots s_{n}$ by generalized contractions only. If $s$ and the $s_{i}$ 's are simple for $1 \leq i \leq n$, then $s_{1} \ldots s_{n} \rightarrow s$ if and only if for some $i, s_{i}$ is an irreducible form of $s_{1} \ldots s_{n}$ and $s_{i} \rightarrow s$.

In linguistic applications, a dictionary lists each word with types belonging to a free pregroup. Then a string of words is a sentence, if one can assign to every word a type from the dictionary such that the concatenation $t_{1} \ldots t_{m}$ of these types has a derivation to a distinguished basic type $s$, called sentence type. In view of Property (2.) above, $t_{1} \ldots t_{m} \rightarrow s$ is equivalent to $t_{1} \ldots t_{m} s^{r} \rightarrow 1$. By the Switching Lemma, a derivation to the empty string can be obtained by generalized contractions only. For example, consider the set of basic types

$B=\left\{\nu_{\mathrm{s}}, \pi_{3 \mathrm{~s}}, o, n_{\mathrm{s}}, c_{\mathrm{s}}, \boldsymbol{s}_{1}, \boldsymbol{s}\right\}$,

where $\nu_{\mathrm{s}} \rightarrow \pi_{3 \mathrm{~s}}, \nu_{\mathrm{s}} \rightarrow o, n_{\mathrm{s}} \rightarrow \pi_{3 \mathrm{~s}}, n_{\mathrm{s}} \rightarrow o, \boldsymbol{s}_{1} \rightarrow \boldsymbol{s}$. Here $\nu_{3}$ is the type for proper names singular, $\pi_{3 \mathrm{~s}}$ for 3 -person singular subjects, $o$ for direct object complements of a verb, $n_{\mathrm{s}}$ for singular noun phrases, $c_{\mathrm{s}}$ for singular common nouns and finally, $s$ and $s_{1}$ are sentence types, the latter for statements in the present tense, the former for statements when the tense does not matter. Then the dictionary may list

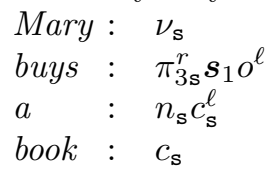

Therefore, the grammar generates the sentence

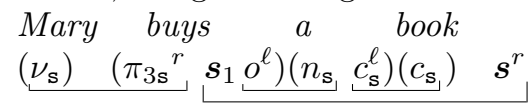

The underlinks indicate the generalized contractions used in the derivation, namely $\nu_{\mathrm{s}} \pi_{3 \mathrm{~s}}^{r} \rightarrow 1, o^{\ell} n_{\mathrm{s}} \rightarrow 1, c_{\mathrm{s}}^{\ell} c_{\mathrm{s}} \rightarrow 1$ and $s_{1} s^{r} \rightarrow 1$. In fact, the underlinks together uniquely determine a derivation to the empty string or more generally, a derivation of $s_{1} \ldots s_{n}$ to a substring $s_{i_{1}} \ldots s_{i_{p}}$ consisting of generalized contractions only. We decompose such a derivation into a geometrical part, consisting of the set of underlinks, and an algebraic part, consisting of the generalized contractions. The geometrical part $R$, also called reduction, has the following definition

Definition 1. A reduction $R$ with respect to $n$ is a set of two-element subsets $\{i, k\} \subseteq\{1, \ldots, n\}$, called underlinks, which satisfy

1) for every $i$ with $1 \leq i \leq n$, there is at most one $k$ such that $\{i, k\} \in R$,

2) if $\{i, k\} \in R$ and $i<l<k$ then there is $i<m<k$ such that $\{l, m\} \in R$.

The algebraic part consists of the generalized contractions

3) $s_{i} s_{k} \rightarrow 1$, for $i<k$ such that $\{i, k\} \in R$ 
Let $\left\{i_{1}, \ldots, i_{p}\right\}$ be the subset of $\{1, \ldots, n\}$ consisting of the elements $i_{l}$ for which there is no $k$ such that $\left\{i_{l}, k\right\}$ belongs to $R$. A reduction $R$ is called a transition from $s_{1} \ldots s_{n}$ to $s_{i_{1}} \ldots s_{i_{p}}$, written

$R: s_{1} \ldots s_{n} \Rightarrow s_{i_{1}} \ldots s_{i_{p}}$

if all three conditions above hold. If the substring $s_{i_{1}} \ldots s_{i_{p}}$ cannot be contracted any further, it is called an irreducible form of $s_{1} \ldots s_{n}$.

The empty string 1 and every simple type is irreducible. A string of simple types has at least one irreducible form. Some strings have several irreducible forms, and there may be different reductions bringing it to the same irreducible form, e.g. the first two reductions below are transitions to the empty string, the third reduction is a transition to the irreducible $a^{r} a$
1) $a^{\ell} a \underline{a^{\ell} a_{1}} a^{r}, a$
2) $\quad \underline{a^{\ell} a} a^{a^{\ell}} \underline{a a^{r}}, a$
3) $\quad a^{\ell} a_{1} a^{\ell} a_{a} a^{r} a$

On the other hand, the same reduction may constitute a transition to the empty string for quite different strings of simple types. For example, assume $a \rightarrow b$ and consider the three different transitions below

$$
b^{\ell} a \underline{b^{\ell} a} a^{r} a_{j}, \quad \underline{b^{\ell} a} b^{\ell} \underline{a} a^{r} a_{j}, \quad c c^{\ell} \underline{c^{r} c^{r r}} c^{r} c^{r} .
$$

The first and the third transition have the same links, the common geometrical part is $R=\{\{1,6\},\{2,5\},\{3,4\}\}$. The second transition, however, has a different reduction $R^{\prime}=\{\{1,2\}\{3,6\}\{4,5\}\}$. This geometrical difference corresponds to different meanings of the same sentence, as illustrated by the examples (1a) and (1b) of the next section.

Using the graphical representation of $\{i, k\} \in R, i<k$, by an underlink

$$
\ldots s_{i} \ldots s_{k} \ldots
$$

we may describe a reduction as a planar graph which has a linearly ordered set of vertices labeled by simple types such that

- there are no loops

- every $i \notin\left\{i_{1}, \ldots, i_{p}\right\}$ is endpoint of exactly one underlink

- underlinks do not cross

- there is no underlink $\{i, k\}$ such that $i \leq i_{l} \leq k$ for $1 \leq l \leq p$.

If $i<k$, we call $i$ or $s_{i}$ the left endpoint and $k$ or $s_{k}$ the right endpoint of the link $\{i, k\}$. If the reduction $R$ is a transition $R: s_{1} \ldots s_{n} \Rightarrow s_{i_{1}} \ldots s_{i_{p}}$, then the iterator of the right endpoint of an underlink is the successor of the iterator of its left endpoint: Indeed, if $\{i, k\} \in R$ and $i<k$, then the algebraic condition $s_{i} s_{k} \rightarrow 1$ implies that $s_{i}=a^{(z)}$ and $s_{k}=b^{(z+1)}$ for some integer $z$ and basic types $a$ and $b$.

Finally, we remark that an arbitrary transition can always be obtained as the union of transitions to the empty string. Indeed, let $R_{l}, 0 \leq l \leq p$, be 
reductions such that $R_{0}: s_{1} \ldots s_{i_{1}-1} \Rightarrow 1, R_{l}: s_{i_{l}+1} \ldots s_{i_{l+1}-1} \Rightarrow 1$ for $1 \leq l<p$, and $R_{p}: s_{i_{p}+1} \ldots s_{n} \Rightarrow 1$. Then the union

$$
R=\bigcup\left\{R_{l}: 0 \leq l \leq p\right\}
$$

is a reduction such that $R: s_{1} \ldots s_{n} \Rightarrow s_{i_{1}} \ldots s_{i_{p}}$.

\section{Coordinate Structures and Unbounded De- pendencies}

We illustrate parsing with pregoup grammars by a few examples on coordination and unbounded dependencies involving the relative pronoun. The semantical dependencies are expressed by (multi)links. Consider the dictionary

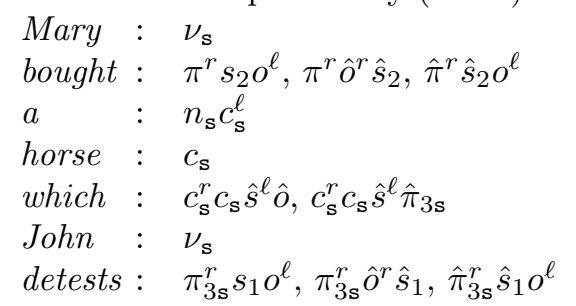

Recall that $o$ is the type of a direct object complement, $\pi_{3 \mathrm{~s}}$ stands for the subject third person singular and $\pi$ for the subject if the person and number do not matter. The dictionary lists three types for the definite verb form. This reflects the grammatical distinction between statements and relative clauses. In particular, the basic type $\hat{s}_{1}$ is the sentence type for relative clauses in the present and $\hat{s}$ the type for relative clauses if the tense does not matter. The other new basic types are used as dummies, namely $\hat{o}$ for a direct object complement, $\hat{\pi}_{3 \mathrm{~s}}$ for a third person subject in the singular, $\hat{\pi}$ if the number and person do not matter. The set of basic types occurring in the dictionary is where

$$
B=\left\{s, s_{1}, s_{2}, \pi_{3 \mathbf{s}}, \pi, o, \nu_{\mathbf{s}}, n_{\mathbf{s}}, c_{\mathbf{s}}, \hat{s}_{1}, \hat{s}, \hat{\pi}_{3 \mathbf{s}}, \hat{\pi}, \hat{o}\right\}
$$

$$
\nu_{\mathrm{s}} \rightarrow \pi_{3 \mathrm{~s}}, n_{\mathrm{s}} \rightarrow \pi_{3 \mathrm{~s}}, \nu_{\mathrm{s}} \rightarrow o, n_{\mathrm{s}} \rightarrow o, \pi_{3 \mathrm{~s}} \rightarrow \pi, s_{1} \rightarrow s, s_{2} \rightarrow s, \hat{s}_{1} \rightarrow \hat{s} .
$$

Then we use different type assignments for the relative pronoun which, according to its role as an object complement or subject in the relative clause.

Example I

$$
\begin{aligned}
& \text { Mary bought a horse which John detests }
\end{aligned}
$$

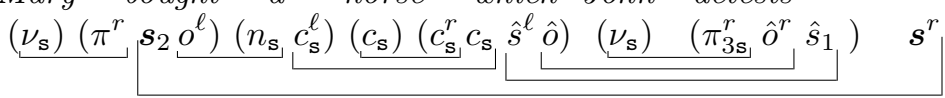

$$
\begin{aligned}
& \text { Mary bought a horse which detests John }
\end{aligned}
$$

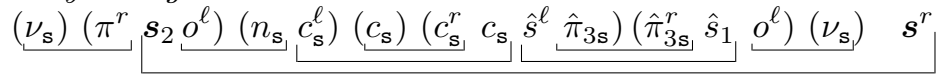

The two reductions above differ only by the links whose left endpoint is under which or later. This becomes more evident with the following abbreviation, called a multilink:

$\underline{Y X^{r}}$ for $t_{1} \ldots t_{p} s_{p}^{r} \ldots s_{1}^{r}$, where $X=s_{1} \ldots s_{p}, \quad Y=t_{1} \ldots t_{p}$. 
Replacing the two links under $\hat{s}^{\ell} \hat{o} \ldots \hat{o}^{r} \hat{s}_{1}$ by a multilink and omitting the types in the first example, we get the dependencies

$$
\text { which John detests }
$$

$$
\cdots
$$

giving the correct the semantical analysis of the relative clause. Applying the same simplifications to the second example, we obtain the dependencies

$$
\text { ...Lhich detests John }
$$

Dummies are entities not explicitly named in a sentence but are implied by the statement. Their syntactical role is to fill what [Gazdar] calls a hole. It is pointed out in $[$ loc. cit.] that holes have a syntactic category. In pregroup grammars, the hole is marked by a right or left adjoint of a dummy type and therefore also has a syntactic representation. We take advantage of this fact when analysing coordinate structures conjoined by and. The sample sentences are adapted from [loc. cit.].

$$
\begin{array}{ll}
\text { and }: & x^{r} x x^{\ell}, x=\hat{o}^{r} \hat{s}_{1} \\
\text { and }: & x^{r} x x^{\ell}, x=\hat{\pi}_{3 \mathrm{~s}}^{r} \hat{s}_{1}
\end{array}
$$

Consider the case where the hole is the object complement of the verb in the relative clause, i.e.

$$
x=\hat{o}^{r} \hat{s}_{1} \text {. }
$$

Let $y=\hat{o}^{r} \hat{s}$. Then

detests, loves : $\pi_{3 \mathrm{~s}}^{r} x$ and

which : $c_{\mathrm{s}}^{r} c_{\mathrm{s}} y^{\ell}$.

It follows that $x \rightarrow y$ and we find the following reduction

Mary bought a horse which John detests and Jo loves

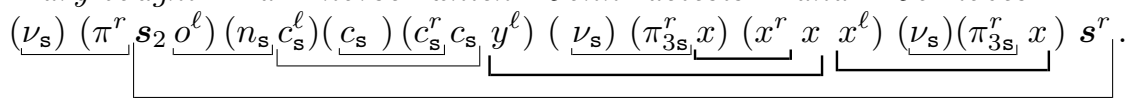

The conjunction and distributes the holes both times to the position of the second argument of the relation expressed by the verb. This position corresponds by convention to the direct object of the verb:

$$
\begin{aligned}
& \text { horse which John detests and Jo loves }
\end{aligned}
$$

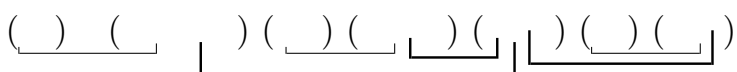

Next, consider the case where the hole is the subject of the relative clause:

$$
x=\hat{\pi}_{3 \mathrm{~s}}^{r} \hat{s}_{1}, y=\hat{\pi}_{3 \mathrm{~s}}^{r} \hat{s} .
$$

Then

detests, loves : $x o^{\ell}$ and which $: c_{\mathrm{s}}^{r} c_{\mathrm{s}} y^{\ell}$

As before, we find $x \rightarrow y$ and with this construct the reduction of the sentence

Mary bought a horse which detests John and loves Jo

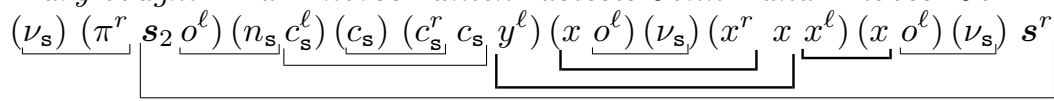

This time, the holes are distributed by the conjunction and to the first argument place of the verb, i.e. to the position of the subject: 
which detests John and loves Jo

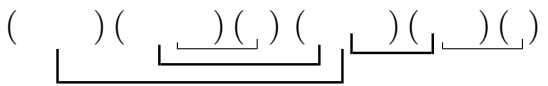

However the two different kinds of holes cannot be conjoined:

* Mary bought a horse which John detests and loves Jo

For a proof, it suffices to consider every type assignment from our dictionary and verify that it has no reduction to the sentence type. Therefore which cannot be both subject and object of verb phrases constituting the relative clause.

\section{Lazy Parsing}

We give a 'lazy' recognition and parsing algorithm and define sufficient conditions on the dictionaries for which it is linear and complete.

In the following, the partially ordered set $B$ and the free pregroup $P(B)$ generated by $B$ are fixed. As usual, a dictionary $\mathcal{D}$ over $B$ for a set of words $V$ is a map from $V$ to the set of subsets of $P(B)$. Instead of $T_{l} \in \mathcal{D}\left(v_{l}\right)$ we may write $v_{l}: T_{l}$ and call it a lexical entry. We distinguish a basic type $s$, the so-called sentence type. A string of types $T_{1} \ldots T_{n}$ is called a type assignment for $v_{1} \ldots v_{n}$ if $v_{l}: T_{l}$ is a lexical entry for $1 \leq l \leq n$. A parsing of a string $v_{1} \ldots v_{n}$ consists of a type assignment $T_{l} \in \mathcal{D}\left(v_{l}\right)$ and a reduction of $T_{1} \ldots T_{n}$ to $s$.

We begin by describing an algorithm which combines the search for a reduction with type assignment. It processes by stages, reading the string of words $v_{1} \ldots v_{n}$ from left to right. At each stage, it either chooses a type for the word under examination or processes the assigned type by reading its simple types from left to right. The result is a reduction of the type processed so far to an irreducible type.

The set of stages associated to $v_{1} \ldots v_{n}$ consists of an initial stage $s_{\text {in }}$ and of triples $s=\left(l, T_{1} \ldots T_{l}, p\right)$ where

$l$ is the number of the word $v_{l}$ being processed

$T_{k}=a_{k 1} \ldots a_{k q_{k}}$ in $\mathcal{D}\left(v_{k}\right), 1 \leq k \leq l$, a type assignment for $v_{1} \ldots v_{l}$

$p$ a position, $0 \leq p \leq q_{l}$.

The stages are partially ordered as follows

$s_{\text {in }}<s$ for all $s$

$\left(l, T_{1} \ldots T_{l}, p\right) \leq\left(l^{\prime}, T_{1}^{\prime} \ldots T_{l^{\prime}}^{\prime}, p^{\prime}\right) \Leftrightarrow l \leq l^{\prime}, T_{k}=T_{k}^{\prime}$ for $1 \leq k \leq l, p \leq p^{\prime}$.

We remark that all non-initial stages $s$ have a unique immediate predecessor, which we denote by $s-1$, i.e.

$$
\left(l, T_{1} \ldots T_{l}, p\right)-1= \begin{cases}\left(l, T_{1} \ldots T_{l}, p-1\right), & \text { if } 1 \leq p \\ \left(l-1, T_{1} \ldots T_{l-1}, q_{l-1}\right) & \text { if } p=0 \text { and } l>1 \\ s_{\text {in }}, & \text { if } p=0 \text { and } l=1\end{cases}
$$

It follows that the set of stages smaller than or equal to a given stage $s$ is totally ordered.

This total order can be used to control the way how the algorithm moves through the stages and define the actual position $p(s)$ and the type $a_{p(s)}$ read 
at this position. At the initial stage $p\left(s_{i n}\right)=0, a_{0}=1$. A stage of the form $\left(l, T_{1} \ldots T_{l}, 0\right), 1 \leq l \leq n$, is called a downloading stage and serves to choose a type $T_{l} \in \mathcal{D}\left(v_{l}\right)$ as soon as the word $v_{l}$ has been given. At a downloading stage $s=\left(l, T_{1} \ldots T_{l}, 0\right)$, the examined position remains unchanged

$$
p(s)=p(s-1)=q_{1}+\cdots+q_{l-1}+0 .
$$

After downloading, the string of simple types $T_{l}$ is read from left to right. Each stage which is not initial and not downloading is called a testing stage. To reach the testing stage $s=\left(l, T_{1} \ldots T_{l}, p\right), p \geq 1$, the preceding position $p(s-1)$ is incremented by 1 :

$$
p(s)=p(s-1)+1=q_{1}+\cdots+q_{l-1}+p .
$$

It follows that the simple type occupying this position satisfies

$$
a_{p(s)}=a_{l p} .
$$

The type processed at stage $s=\left(l, T_{1} \ldots T_{l}, p\right)$ can be defined inductively by

$$
\begin{array}{llll}
T\left(s_{\text {in }}\right) & =1 & & \\
T(s) & =T(s-1) & & a_{0} \\
T(s) & =T(s-1) a_{p(s)} \ldots T_{l-1} & & \text { if } 0=p \\
& =T_{1} \ldots T_{l-1} a_{l 1} \ldots a_{l p} & & \text { if } 0<p
\end{array}
$$

More generally, for every $i$ such that $1 \leq i \leq p(s)$ there are a unique $k$ and a unique $p^{\prime}$ such that $1 \leq k \leq l, 1 \leq p^{\prime} \leq q_{k}, i=q_{1}+\cdots+q_{k-1}+p^{\prime}$ and

$$
a_{i}=a_{k p^{\prime}}
$$

The simple type $a_{p(s)}$ is tested for generalized contraction with the last not contracted type in the string. This test can be done in one time unit by accessing the partial order relation on the set of basic types. If it fails, $p(s)$ is added on the top of the stack indicating that $a_{p(s)}$ is the latest not (yet) contracted type. The other data remain unchanged. If the test succeeds, the stack is popped and the link consisting of the contracting positions is added to the reduction computed so far.

A stack $S(s)$ constructed by this algorithm at stage $s$ is an ordered pair $\left\langle S^{\prime}, i\right\rangle$ where $i$ is a non-negative integer and $S^{\prime}$ is either the empty stack $\varnothing$ or the stack of a preceding stage. As the test is only performed for non-initial and non-downloading stages, all positions $i$ stored in the stack at stage $s$ correspond to a unique stage $s^{\prime} \leq s$ for which $a_{p\left(s^{\prime}\right)}=i$. The functions top and pop send a stack $\left\langle S^{\prime}, i\right\rangle$ to its top $i$ and its tail $S^{\prime}$ respectively. They are undefined for the empty stack. A stack of positions defines a substring of $a_{1} \ldots a_{n}$ as follows

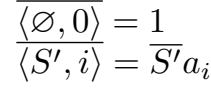

\section{Definition 2. Lazy Parsing Algorithm}

$\boldsymbol{\nabla}$ At the initial stage, let

$$
S\left(s_{i n}\right)=\langle\varnothing, 0\rangle, R\left(s_{\text {in }}\right)=\emptyset
$$


V At a downloading stage $s=\left(l, T_{1} \ldots T_{l}, 0\right)$, the stack and reduction remain unchanged

$$
S(s)=S(s-1), R(s)=R(s-1)
$$

$\boldsymbol{\nabla}$ If $s=\left(l, T_{1} \ldots T_{l}, p\right)$ is not downloading and not initial, let $t(s-1)=\operatorname{top}(S(s-$ 1)). Then

$$
\begin{aligned}
S(s) & = \begin{cases}\operatorname{pop}(S(s-1)), & \text { if } a_{t(s-1)} a_{p(s)} \rightarrow 1 \\
\langle S(s-1), p(s)\rangle, & \text { else }\end{cases} \\
R(s) & =\left\{\begin{array}{ll}
R(s-1) \cup\{\{t(s-1), p(s)\}\}, & \text { if } a_{t(s-1)} a_{p(s)} \rightarrow 1 \\
R(s-1), & \text { else }
\end{array} .\right.
\end{aligned}
$$

The stack can increase and decrease during processing, but it will never get empty. Indeed, if $S(s-1)=\langle\varnothing, 0\rangle$ then the test will fail, as $a_{0} a_{p(s)}=a_{p(s)} \nrightarrow 1$. Hence $S(s)=\langle\langle\varnothing, 0\rangle, p(s)\rangle$. Note also that the entries of the stack form a strictly increasing string of integers between 0 and $p(s)$. Moreover, the set $R$ computed at stage $s$ is a reduction of the string processed so far to an irreducible string, which is defined by the stack $S$ :

Theorem 3 (Irreducible From). For every stage $s=\left(l, T_{1} \ldots T_{l}, p\right)$, the string $\overline{S(s)}$ associated to the stack $S(s)$ is an irreducible substring of $T(s)$ and $R(s)$ is a transition from $T(s)$ to $\overline{S(s)}$.

Proof: Use induction on the linearly ordered set of the predecessors of $s$. Clearly, the property holds for the initial stage. Now assume that the property holds up to $s-1$. If $s$ is a downloading stage, the property follows immediately from the induction hypothesis. If $s$ is a testing stage, let $t(s)=\operatorname{top}(S(s))$ and decompose the processed string $T(s)=a_{1} \ldots a_{p(s)}$ into an initial and a terminal segment

$$
a_{1} \ldots a_{p(s)}=\left(a_{1} \ldots a_{t(s)}\right)\left(a_{t(s)+1} \ldots a_{p(s)}\right)
$$

and show that $R(s)$ is the union of two reductions

(1) $R^{\prime}(s): a_{1} \ldots a_{t(s)} \Rightarrow \overline{S(s)}$

(2) $\quad R^{\prime \prime}(s): a_{t(s)+1} \ldots a_{p(s)} \Rightarrow 1$.

For that purpose, distinguish two cases:

Case I : $a_{t(s-1)} a_{p(s)} \not \rightarrow 1$.

Then $t(s)=p(s), S(s)=\langle S(s-1), p(s)\rangle$ and $R(s)=R(s-1)$ and $\overline{S(s)}=$ $\overline{S(s-1)} a_{t(s)}$. Then $a_{t(s)+1} \ldots a_{p(s)}$ is the empty string and the restriction $R^{\prime \prime}(s)$ of $R(s)$ to this string is the empty set. Therefore (2) holds trivially.

Next, from $a_{t(s-1)} a_{t(s)} \nrightarrow 1$ and the induction hypothesis follows that $\overline{S(s)}=$ $\overline{S(s-1)} a_{t(s)}$ is an irreducible form of $a_{1} \ldots a_{p(s)}=\left(a_{1} \ldots a_{p(s)-1}\right) a_{p(s)}$. Recall that $p(s-1)=p(s)-1$ and that $R(s-1): a_{1} \ldots a_{p(s-1)} \Rightarrow \overline{S(s-1)}$ by induction hypothesis. Hence $R(s): a_{1} \ldots a_{p(s)} \Rightarrow \overline{S(s-1)} a_{p(s)}=\overline{S(s)}$. As $R^{\prime}(s)=R(s),(1)$ holds.

Case (II) $: a_{t(s-1)} a_{p(s)} \rightarrow 1$.

In this case, 


$$
S(s-1)=\left\langle S^{\prime}, t(s-1)\right\rangle
$$

where $S^{\prime}$ is the stack computed at some stage $s^{\prime}$ preceding $s-1$. Moreover,

$$
S(s)=\operatorname{pop}(S(s-1))=S^{\prime}
$$

and

$$
R(s)=R(s-1) \cup\{t(s-1), p(s)\} .
$$

Then $t(s)=\operatorname{top}\left(S^{\prime}\right), \overline{S(s)}=\overline{S^{\prime}}$ and $\overline{S(s-1)}=\overline{S^{\prime}} a_{t(s-1)}=\overline{S(s)} a_{t(s-1)}$. By induction hypothesis

(1) $\quad R^{\prime}(s-1): a_{1} \ldots a_{t(s-1)} \Rightarrow \overline{S(s-1)}$

(2) $\quad R^{\prime \prime}(s-1): a_{t(s-1)+1} \ldots a_{p(s-1)} \Rightarrow 1$.

Now decompose $a_{1} \ldots a_{p(s)}$ as follows

$$
a_{1} \ldots a_{p(s)}=
$$

$\left(a_{1} \ldots a_{t(s)}\right)\left(a_{t(s)+1} \ldots a_{t(s-1)-1}\right) a_{t(s-1)}\left(a_{t(s-1)+1} \ldots a_{p(s-1)}\right) a_{p(s)}$.

Note that $R^{\prime}(s)$, the restriction of $R(s)$ to $\{1, \ldots, t(s)\}$, is also the restriction of $R(s-1)$ to $\{1, \ldots, t(s)\}$. Hence

$$
R^{\prime}(s): a_{1} \ldots a_{t(s)} \Rightarrow \overline{S(s)} .
$$

Next, let $R^{*}(s)$ be the restriction of $R(s-1)$ to $\{t(s)+1, \ldots, t(s-1)-1\}$. It reduces the segment $a_{t(s)+1} \ldots a_{t(s-1)-1}$ between two consecutive positions of $\overline{S(s-1)}$ to 1 . Finally, the restriction of $R(s)$ to $\{t(s)+1, \ldots, p(s)\}$ is

$$
\left.R^{\prime \prime}(s)=R^{*}(s) \cup R^{\prime \prime}(s-1) \cup\{t(s-1), p(s)\}\right) .
$$

Hence

$$
R^{\prime \prime}(s):\left(a_{t(s)+1} \ldots a_{t(s-1)-1}\right)\left(a_{t(s-1)}\left(a_{t(s-1)+1} \ldots a_{p(s-1)}\right) a_{p(s)}\right) \Rightarrow 1 .
$$

We remark that the number of steps necessary to compute $S(s)$ from its predecessor $S(s-1)$ is bounded by a constant which depends only of the dictionary. Hence we have the following property

Corollary 4. For every stage $s=\left(n, T_{1} \ldots T_{n}, q_{n}\right)$, Lazy Parsing computes the irreducible form $\overline{S(s)}$ and the reduction $R(s): T_{1} \ldots T_{n} \rightarrow \overline{S(s)}$ in time proportional to the length of $T_{1} \ldots T_{n}$.

Note that Lazy Parsing also works on infinite dictionaries. Consider the identity map on the set of simple types $\Sigma$, i.e. the dictionary with the entries $t: t$, for $t \in \Sigma$. The set of stages associated to the string $t_{1} \ldots t_{n}$ is $\{1, \ldots, n\}$ and Lazy Parsing produces a derivation of $t_{1} \ldots t_{n}$ to one of its irreducible forms.

Backward Lazy Parsing, that is to say reading a string of words from right to left, is defined similarly. In particular, the positions stored in the stack are decreasing and the test for contraction is $a_{p(s)} a_{t(s-1)} \rightarrow 1$. The appropriate variant of the preceding theorem then holds for Backward Lazy Parsing. In general, the computed irreducible form may be different from that computed by 'forward' lazy parsing. For example, backward parsing finds the second reduction of (I) in Section (2) and forward parsing the third. 
If the computed irreducible form happens to be the sentence type, the algorithm gives a parsing of the sentence. If this is not the case, we cannot conclude in general that $T_{1} \ldots T_{n}$ has no reductions to the sentence type. Hence, the algorithm is not complete unless we impose some conditions on the dictionary. The rest of this section is devoted to define a class of pregroup grammars for which the Parsing Algorithm is complete. An even more comprehensive class of pregroup grammars can also be parsed in linear time by a slight variant of our algorithm, as we shall see in the next section.

Definition 5. (Complexity:)

A connected component $\mathcal{C}$ of basic types has complexity $K \geq 0$, if there is an integer $u$ satisfying the following two conditions

whenever $b \in \mathcal{C}$ and $b^{(z)}$ occurs in $\mathcal{D}$, then $u \leq z \leq u+K$

there are $b_{i} \in \mathcal{C}$ for which $b_{i}{ }^{(u+i)}$ occurs in $\mathcal{D}$, for $0 \leq i \leq K$.

A simple type $t=a^{(z)}$ has complexity $K$ if $a$ belongs to a connected component of complexity less than or equal to $K$. A string of simple types has complexity $K$ if every simple type in the string has complexity $K$. A grammar is of complexity $K$ if every type in its dictionary has complexity $K$.

Our sample dictionary of Section (3) has complexity 2. Indeed, the simple types occurring in the dictionary are either basic or right or left adjoints of basic types. Without the words which and and, it would be of complexity 1 .

Grammars of complexity 1 are interesting because of their algorithmic properties, but they are not expressive enough. The next class, that of complexity 2 , is as expressive as the whole class of pregroup grammars. Indeed, every pregroup grammar is strongly equivalent to a dictionary using only basic types, right adjoints of basic types and left adjoints of basic types, see [Preller 07]. Here strongly equivalent means not only that the grammars generate the same sentences but also that a reduction of a type-assignment in one grammar is also a reduction for some type assignment in the other grammar. Hence the links under the words are the same in both grammars.

We will show that the Lazy Parsing Algorithm is complete for dictionaries of complexity 1 and indeed for an even larger class, the linear dictionaries.

Definition 6. (Critical types, critical triples and linear types)

A simple type $t=c^{(z+1)}$ is critical if there are simple types $a, b$ in the connected component of $c$ such that $a^{(z-1)}$ and $b^{(z)}$ occur in $\mathcal{D}$.

A simple type $t=b^{(z)}$ occurring in $\mathcal{D}$ is said to be right-only if $c^{(z+1)}$ does not occur in $\mathcal{D}$ for all $c$ in the connected component of $b$. The notion of left-only is defined similarly.

Let $T=t_{1} \ldots t_{n}$ be a string of simple types and assume that $1 \leq i<j<k \leq n$. We say that $t_{i}, t_{j}, t_{k}$ is a critical triple if

$$
\begin{aligned}
& t_{i} t_{j} \rightarrow 1, t_{j} t_{k} \rightarrow 1, \\
& t_{i+1} \ldots t_{j-1} \rightarrow 1, t_{j+1} \ldots t_{k-1} \rightarrow 1 .
\end{aligned}
$$

A string of simple types without critical triples is called linear. A dictionary is linear, if all type assignments with a reduction to the sentence type are linear. 
Compare the notion of a critical triple with that of a critical type. The former is relative to a given string of simple types, whereas the latter depends only of the dictionary. The last of a critical triple is necessarily a critical type. If the dictionary has complexity 2 , the last of a critical triple $\ldots t_{i} \ldots t_{j} \ldots t_{k} \ldots$ is right-only and the first left-only. A string is necessarily linear if no critical type occurs in it. Hence dictionaries of complexity 1 are linear.

Lemma 7 (Uniqueness of Links in Linear Strings). Suppose $t_{1} \ldots t_{m}$ is a linear string of simple types. Then for every position $k$ there is at most one $i$ satisfying $i<k, t_{i} t_{k} \rightarrow 1$ and $t_{i+1} \ldots t_{k-1} \rightarrow 1$, or

$$
i>k, t_{k} t_{i} \rightarrow 1 \text { and } t_{k+1} \ldots t_{i-1} \rightarrow 1 .
$$

Proof: Note that $k$ can not be both the right endpoint of a link $\{i, k\}$ and the left endpoint of a link $\{k, j\}$, because if this was the case, the string would have the critical triple $t_{i} \ldots t_{k} \ldots t_{j}$. Show that $k$ can not be endpoint of two different links using induction on the length $m$ of the longer link, i.e. $m=$ $\max \{|k-i|,|k-j|\}$. If $m=1, t_{k}$ would be linked to $t_{k-1}$ and to $t_{k+1}$, which is impossible as we just saw. If $m=2$ we would have a link $\{i, i+2\}$ with $t_{i+1} \rightarrow 1$, which is impossible for a simple type. Suppose the property holds up to $m-1$, with $3 \leq m$. Assume first that $t_{k}$ is right endpoint of two different links, i.e. that for some $i, j \geq 1$

$$
\begin{aligned}
i<j & <k \\
t_{i} t_{k} & \rightarrow 1 \\
t_{i+1} \ldots t_{k-1} & \rightarrow 1 \\
t_{j} t_{k} & \rightarrow 1 \\
t_{j+1} \ldots t_{k-1} & \rightarrow 1
\end{aligned}
$$

By hypothesis, there are a reductions $R$ and $R^{*}$ such that $R: t_{i} \ldots t_{k} \Rightarrow 1$ and $\{i, k\} \in R$ and $R^{*}: t_{j} \ldots t_{k} \Rightarrow 1$ and $\{j, k\} \in R^{*}$. Using underbrackets for the links of $R$ and underbraces for the links of $R^{*}$, the situation is represented by the graph

$$
\ldots \underbrace{t_{i} \ldots \underbrace{t_{j} \ldots \quad \ldots \quad \ldots t_{k}} \cdots} \cdots
$$

Then $R$ links $j$ to some position $k^{\prime}$ with $i+1 \leq k^{\prime} \leq k-1$. Note that $k^{\prime}$ is necessarily to the right of $j$, as otherwise the string would have the critical triple

$$
\ldots t_{k^{\prime}} \ldots \underbrace{t_{j} \ldots t_{k}} \ldots, \text { which is impossible. }
$$

Hence, $j<k^{\prime}<k$ and

$$
\ldots t_{i} \ldots \underbrace{t_{j} \ldots t_{k^{\prime}}} \ldots t_{k} \ldots .
$$

Then $t_{j}$ would be left endpoint of two different links. As $|j-k|<|i-k|$, this contradicts the induction hypothesis. The case where $t_{k}$ is left endpoint is similar. 
This local property of unique links has useful global consequences. The first one is the following Lemma

Lemma 8 (Uniqueness of Reductions of Linear Strings). Every linear string of simple types $t_{1} \ldots t_{m}$ has a unique irreducible form $t_{i_{1}} \ldots t_{i_{p}}$ and a unique reduction $R$ to that irreducible form. This reduction is computed by Lazy Parsing

Proof: If $t_{1} \ldots t_{m}$ is irreducible, the property holds trivially. Else, note that in all reductions to an irreducible form, the links with neighboring endpoints must be identical. Indeed, let $R$ and $R^{\prime}$ be reductions such that $R: t_{1} \ldots t_{m} \Rightarrow$ $t_{i_{1}} \ldots t_{i_{p}}$ and $R^{\prime}: t_{1} \ldots t_{m} \Rightarrow t_{j_{1}} \ldots t_{j_{q}}$ with $t_{i_{1}} \ldots t_{i_{p}}$ and $t_{j_{1}} \ldots t_{j_{q}}$ irreducible. Assume $\{k-1, k\} \in R$. As $t_{k-1} t_{k} \rightarrow 1$, one of $k-1$ or $k$ must belong to a link in $R^{\prime}$. If it is $k$, it can not be the left endpoint of a link $\{k, i\} \in R^{\prime}$, because otherwise the string would have a critical triple, contradicting linearity. Hence $R^{\prime}$ can link $k$ only to some position $i<k$. By the preceding lemma, it follows that $i=k-1$. Similarly, if it is $k-1$ that is linked in $R^{\prime}$ to some position, the latter is necessarily the position $k$. Hence $R$ and $R^{\prime}$ coincide on all links with neighboring endpoints. Omitting these links from $R$ and $R^{\prime}$ and the corresponding segments $t_{k-1} t_{k}$ from $t_{1} \ldots t_{m}$, the new reductions are reductions of the new string to the same irreducible forms $t_{i_{1}} \ldots t_{i_{p}}$ and $t_{j_{1}} \ldots t_{j_{q}}$ respectively. It follows by induction hypothesis, that the same positions are linked by $R$ and by $R^{\prime}$. But then the positions that are not linked also coincide in both reductions. Therefore $p=q, i_{k}=j_{k}$, for $1 \leq k \leq p$ and $t_{i_{1}} \ldots t_{i_{p}}=$ $t_{j_{1}} \ldots t_{j_{q}}$.

Theorem 9 (Linear Completeness). A string of words from a linear dictionary $v_{1} \ldots v_{n}$ is a sentence if and only if at some final stage $s=$ $\left(n, T_{1} \ldots T_{n}, q_{n}\right)$, the reduction $R(s)$ reduces $T_{1} \ldots T_{n}$ to the sentence type. Moreover, for a given final stage, the reduction has been computed by Lazy Parsing in time proportional to the length of the corresponding type assignment.

Proof: The last assertion follows from the fact that the definition of $R(s)$ only involves the predecessors of $s$ and that for each stage the number of computation steps is constant. The rest is a straightforward consequence of Theorem 3 and Lemma 8.

Hence Lazy Parsing finds the unique reduction of a linear string to an irreducible form. The same algorithm does as well for certain strings with critical triples. Consider a string which has all critical types at the end, like

$$
\underline{b \underline{b^{\ell} b} \underline{b^{\ell} b} b^{r} b^{r}} \text {. }
$$

Such a string has at most one reduction to the empty string, which is computed by Lazy Parsing.

Lemma 10 (Quasi-Linearity). Suppose that none of the simple types in $S=$ $s_{1} \ldots s_{n}$ is critical and all of the simple types in $T=t_{1} \ldots t_{m}$ are right-only. Then ST has at most one reduction to the empty string. Moreover, if a reduction to the empty string exists, it is computed by Lazy Parsing. 
Proof: Suppose $R: S T \Rightarrow 1$. Every critical type is a right endpoint and cannot be linked to another critical type. Hence it is linked to some $s_{i}$ with $i \leq n$.

$$
\ldots s_{i_{1}} \ldots \ldots s_{i_{m-1}} \ldots s_{i_{m}} \ldots t_{1} t_{2} \ldots \ldots t_{m}
$$

Omitting these links from $R$, we obtain a reduction $R^{\prime}$ of $S$ to the irreducible string $s_{i_{1}} \ldots s_{i_{m-1}} s_{i_{m}}$. Indeed, the segment between $s_{i_{m}}$ and $t_{1}$ is reduced by $R$ to the empty string, because links do not cross. For the same reason, the segments between two consecutive $s_{i_{k}}$ 's and the initial segment up to $s_{1}$ are reduced to the empty string by $R$ and hence by $R^{\prime}$. Now assume $Q: S T \Rightarrow 1$ is another reduction to the empty string. Then its restriction to $Q^{\prime}$ to $S$ as well is a reduction of $S$ to an irreducible form $s_{j_{1}} \ldots s_{j_{m^{\prime}}}$. By the Linearity Lemma, $S$ has a unique irreducible form and a unique reduction to this irreducible form. Therefore $Q^{\prime}=R^{\prime}, m=m^{\prime}$ and $i_{k}=j_{k}$ for $1 \leq k \leq m$. As links do not cross, it follows that $Q=R$. Finally, as $S$ is linear, Lazy Parsing computes the unique reduction $R^{\prime}: S \Rightarrow s_{i_{1}} \ldots s_{i_{m}}$. The indices $i_{1}, \ldots, i_{m}$ are stored in its stack in increasing order. Thus $i_{m}$ is on the top of the stack after computing at stage $n$. Reading the type $t_{1}$ at the next stage, it will pop $i_{m}$ and add $\left\{i_{m}, n+1\right\}$ to the reduction and so on.

We call a string of the form $S T$ in the lemma above quasi-linear and remark that it can have several irreducible forms. For example, the empty string and $b b^{\ell}$ are two irreducible forms of $b b^{\ell} b b^{\ell} b b^{r}$.

In the next section we relax the restrictive conditions above, motivated by properties of the sample dictionary of Section 3. We also modify the parsing algorithm slightly so that it is complete and linear for the larger class of dictionaries defined by the weaker conditions.

\section{Minimal Reductions}

Dictionaries of complexity 2 may have type assignments with critical triples. Such strings often have different irreducible forms and even for a given irreducible form there may be different reductions to it. The time necessary to find all possible reductions to the sentence type can increase exponentially with the number of words, and this even if the dictionary lists just one type per word. The best we can attempt therefore is to compute one particular reduction to the sentence type per type assignment, provided there is at least one. In this section we single out the minimal reduction always present among all reductions to the sentence type.

The critical triples of our sample dictionary of Section 3 are due to the lexical entries

which : $c_{\mathrm{s}}^{r} c_{\mathrm{s}} \hat{s}^{\ell} \hat{o}, c_{\mathrm{s}}^{r} c_{\mathrm{s}} \hat{s}^{\ell} \hat{\pi}_{3 \mathrm{~s}}$

and : $x^{r} x x^{\ell}$,

A critical type in these entries is followed by its own left adjoint or is in a segment that is followed by its left adjoint. This property is the key to complete linear parsing. 
Definition 11. (Guards) A type $T=X C Y$ is guarded if

- there are no critical types in $X$ nor $Y$

- every simple type in $C$ is critical

- $C^{\ell}$ is the smallest element in its connected component

- $Y=C^{\ell} Y^{\prime}$ for some $Y^{\prime}$.

If $C$ is not empty, the following segment $C^{\ell}$ is called the guard of $C$.

A dictionary $\mathcal{D}$ is guarded if every type $T \in \mathcal{D}(v)$ is guarded for all $v$. A pregroup grammar is guarded if its dictionary is guarded.

Every dictionary of complexity 1 is guarded, because such a dictionary has no critical types. In general, the dictionaries proposed for natural languages so far are guarded or have equivalent guarded dictionaries. At this stage it is not known if guarded grammars are as expressive as the whole class of pregroup grammars.

Our aim is to define a subset of the set of all reductions that is small enough for a slightly amended Lazy Parsing algorithm to compute its members. It also must be big enough so that if a given string has a reduction to the sentence type, one of them is in the subset. We can do this for guarded dictionaries of complexity 2 , for which we define the minimal reductions below.

Definition 12. (Fans:) Consider a reduction $R$ of $t_{1} \ldots t_{n}$. A fan of $R$ is a subset of underlinks $\left\{\left\{i_{p}, k\right\},\left\{i_{p-1}, k+1\right\} \ldots,\left\{i_{1}, k+p-1\right\}\right\}$ such that the right endpoints form a segment.

$$
\ldots \underbrace{}_{i_{1} \ldots \ldots t_{i_{p-1}} \ldots t_{t_{p}} \ldots t_{k}} t_{k+1} \ldots t_{k+p-1} \ldots
$$

A fan is critical, if every right endpoint is a critical type. A guarded fan is a critical fan such that $t_{k+p} \ldots t_{k+2 p-1}$ guards the segment $t_{k} \ldots t_{k+p-1}$ of its right endpoints.

A fan reduces the segments between to left endpoints to the empty type and the same is true for the segment between the last left and the first right endpoint. This follows at once from the following, more general property.

Lemma 13. Fan Lemma Consider the following underlinks

$$
\ldots t_{i_{1}} \Gamma_{1} \ldots \ldots t_{i_{p-1}} \Gamma_{p-1} \underbrace{\Lambda_{1} t_{k+1} \ldots \Lambda_{p-1} t_{k+p-1} \ldots}_{t_{i_{p}} \Theta t_{k}}
$$

of a reduction $R$. Then $R: \Gamma_{i} \Rightarrow 1$ if and only if $R: \Lambda_{p-i} \Rightarrow 1$ for $1 \leq i \leq p-1$. Moreover, $R: \Theta \Rightarrow 1$. In particular, the segments between two consecutive left endpoints of a fan reduce to the empty string.

Proof: The last assertion holds in any reduction. Moreover, a link that has one endpoint in $\Gamma_{i}$ has the other endpoint either in $\Gamma_{i}$ itself or in $\Lambda_{p-i}$. Hence $R: \Lambda_{p-i} \Rightarrow 1$ implies $R: \Gamma_{i} \Rightarrow 1$ and vice versa. 
Definition 14. (Minimal reductions:) Let $t_{1} \ldots t_{n}$ be a string of simple types. The fan

$$
\ldots t_{j_{1}} \ldots \ldots t_{j_{p-1}} \ldots t_{j_{p}} \ldots t_{k} t_{k+1} \ldots t_{k+p-1} .
$$

is shorter than the fan

$$
\ldots t_{i_{1}} \ldots \ldots t_{i_{p-1}} \ldots t_{i_{p}} \ldots t_{k} t_{k+1} \ldots t_{k+p-1} .
$$

if $i_{p}<j_{1}$. A fan is minimal if there is no shorter fan with the same right endpoints.

A reduction of $t_{1} \ldots t_{n}$ to the empty string is minimal if all guarded critical fans are the right endpoint of a minimal fan.

In the case where a minimal fan has just one right endpoint, we call it a minimal link. Note that a minimal reduction may have non-minimal links. For example, consider the reductions

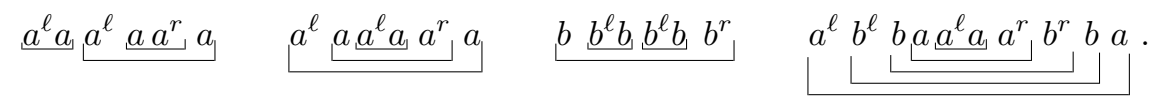

The first reduction is minimal because all its links are minimal. The second is not minimal, it has a guarded non minimal link. The third reduction is also minimal, because its only critical type $b^{r}$ is not guarded and therefore is not required to be minimal. The fourth is again minimal, even though the link through $a^{r}$ is not.

Theorem 15 (Completeness of Minimal Reductions). Assume that $\mathcal{D}$ is a guarded dictionary of complexity 2 . Then for every type assignment $X_{i} \in \mathcal{D}\left(v_{i}\right)$ such that $X_{1} \ldots X_{n} \rightarrow s$ there is a minimal reduction of $X_{1} \ldots X_{n}$ to $s$.

Proof: We must show that whenever $R: X_{1} \ldots X_{n} s^{r}=t_{1} \ldots t_{m} \Rightarrow 1$ is a reduction then there is a minimal reduction $M$ such that $M: t_{1} \ldots t_{m} \Rightarrow 1$. We use induction on the number of critical types in $t_{1} \ldots t_{m}$.

The property is trivially true if there is no critical type. Otherwise, let $t_{k}$ be the leftmost critical type. Note that the string $t_{1} \ldots t_{k-1}$ is linear. As the dictionary is guarded, $t_{k}$ is either the last type in the string or is followed by another critical type or $t_{k+1}$ is the guard of $t_{k}$. In the first case the reduction $R$ is trivially minimal. Now assume that $t_{k+1}$ is the guard of $t_{k}$.

Consider two cases.

Case (i):

The link $\{i, k\}$ is minimal.

Then the restriction $R^{\prime}$ of $R$ to $\{i, \ldots, k\}$ is a reduction $R^{\prime}: t_{i} \ldots t_{k} \Rightarrow 1$. Omit the segment $t_{i} \ldots t_{k}$ from $t_{1} \ldots t_{m}$ and the links with an endpoint in $\{i, \ldots, k\}$ from $R$, obtaining the string $t_{1} \ldots t_{i-1} t_{k+1} \ldots t_{m}$ and a reduction $R_{1}: t_{1} \ldots t_{i-1} t_{k+1} \ldots t_{m} \Rightarrow 1$. As the induction hypothesis applies to $R_{1}$, there 
is a minimal reduction $M^{*}: t_{1} \ldots t_{i-1} t_{k+1} \ldots t_{m} \Rightarrow 1$. Inserting the segment $t_{i} \ldots t_{k}$ with the underlinks of $R^{\prime}$ into $M^{*}$ we obtain a minimal reduction of $t_{1} \ldots t_{m}$ to the empty type.

Case (ii): The link $\{i, k\}$ is not minimal and $t_{k+1}$ guards $t_{k}$.

Let $j$ be the rightmost position $i<j<k$ such that there is a reduction $R^{*}$ satisfying

$$
\begin{aligned}
\{j, k\} & \in R^{*} \\
t_{j} t_{k} & \rightarrow 1 \\
R^{*}: \Theta=t_{j+1} \ldots t_{k-1} & \Rightarrow 1
\end{aligned}
$$

It suffices to construct a reduction $R^{\prime}: t_{1} \ldots t_{m} \Rightarrow 1$ linking $j$ and $k$ and conclude by Case(i).

First we remark that $R$ coincides with $R^{*}$ on $\Theta$. Indeed, $t_{i+1} \ldots t_{k-1}$ is linear and both $R$ and $R^{*}$ link a type in $\Theta$ to a type in $t_{i+1} \ldots t_{k-1}$. Therefore they coincide in $\Theta$ by the Linearity Lemma 7. Hence

$$
R: \Theta=t_{j+1} \ldots t_{k-1} \Rightarrow 1
$$

It follows that $R$ links $j$ to a position $h$ between $i$ and $j$. Hence

$$
\begin{gathered}
\{h, j\} \in R, h<j, t_{h} t_{j} \rightarrow 1 \\
R: \Delta=t_{h+1} \ldots t_{j-1} \Rightarrow 1 .
\end{gathered}
$$

Then $R$ has the underlinks

$$
t_{i} \Gamma t_{h} \Delta t_{j} \Theta t_{k}
$$

As $R$ links each position in $\Theta$ to another position in $\Theta$, the same holds for $\Gamma$, i.e.

$$
R: \Gamma=t_{i+1} \ldots t_{h-1} \Rightarrow 1 \text {. }
$$

Let $l$ be the position linked to $k+1$ in $R$. Note that either $l>k+1$ or $l<i$. If $l>k+1$, i.e. if

$$
R \quad \ldots t_{i} \Gamma t_{h} \Delta t_{j} \Theta t_{k} t_{k+1} \ldots t_{l} \ldots
$$

construct a new reduction $R^{\prime}: t_{1} \ldots t_{m} \Rightarrow 1$ by omitting the links $\{i, k\},\{h, j\}$ and $\{k+1, l\}$ from $R$ and adding the links $\{j, k\},\{h, k+1\}$ and $\{i, l\}$ instead. The other links remain unchanged. Then $R^{\prime}$ looks like this

$$
R^{\prime} \quad \ldots t_{i} \Gamma t_{h} \Delta t_{j} \Theta t_{k} t_{k+1} \ldots t_{l} \ldots
$$

If $l<i$ i.e. if

$$
R \quad \ldots t_{l} \ldots t_{i} \Gamma t_{h} \Delta t_{j} \Theta t_{k} t_{k+1} \ldots
$$


let again $R^{\prime}$ is obtained from $R$ by omitting the links $\{i, k\},\{h, j\}$ and $\{k+1, l\}$ from $R$ and adding the links $\{j, k\},\{h, k+1\}$ and $\{i, l\}$ instead. Now, $R^{\prime}$ looks like this

$$
R^{\prime} \quad \ldots t_{l} \ldots t_{i} \Gamma t_{h} \Delta t_{j} \Theta t_{k} t_{k+1} \cdots
$$

To see that $R^{\prime}: t_{1} \ldots t_{m} \Rightarrow 1$, note that the geographical conditions are satisfied because of V, VII and IX. Moreover, the algebraic conditions obviously hold for the unchanged links and for the minimal link $\{j, k\}$. They also hold for the new links. Indeed, $t_{k+1}=t_{k}^{\ell}$ is the smallest element in its connected component by assumption. Hence whenever $t t_{k} \rightarrow 1$ then $t=t_{k+1}$.

Remains the case where $t_{k+1}$ is another critical type, i.e. where the leftmost critical fan has several right endpoints. We only consider the case where $R$ is not minimal. Let $R^{*}$ be a reduction that defines a shorter fan than $R$.

$R^{*}$

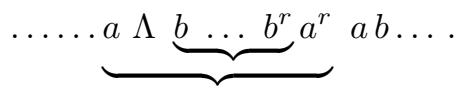

Let $\Lambda_{q}$ be the intermediary segment of any two successive left endpoints of $R^{*}$. Using linearity of the string before the first critical type we show that $R$ coincides with $R^{*}$ on $\Lambda_{q}$ and cancels the left endpoints of $R^{*}$ to the right of its own left endpoints

$$
R \quad \ldots a \ldots b \ldots b^{\ell} \Gamma \underline{a}^{\ell} \ldots a_{\Lambda} \Lambda b_{1} \ldots b^{r} a^{r} a b \ldots
$$

From this follows by the Fan Lemma,

$$
R: \Lambda_{q} \Rightarrow 1, \quad R: \Gamma_{q} \Rightarrow 1
$$

Now we construct $R^{\prime}$ as above. This ends the proof.

\section{Minimal Parsing}

Recall that a string with $n$ critical types may have up to to $2^{n}$ different reductions. Hence looking for only one of them should diminish the work considerably. In fact, Lazy Parsing can be amended to find such a minimal reduction keeping its run-time still linear. We formulate the relevant properties of Lazy Parsing in the next two lemmas. For a fixed string of simple types $T=t_{1} \ldots t_{n}$, the stages of Lazy Parsing identify with the positions $\{0,1, \ldots, n\}$, where 0 stand for the initial stage. Hence, Lazy Parsing processes $t_{i}$ at stage $i$.

Lemma 16. Assume that the reduction $R: T=t_{1} \ldots t_{n} \Rightarrow 1$ includes the critical fan

$$
\ldots t_{j_{1}} \ldots \ldots L_{j_{p-1}} \ldots t_{j_{p}} \ldots t_{k} t_{k+1} \ldots t_{k+p-1} \ldots
$$

If $T$ is of complexity 2 then $t_{j_{1}} \ldots t_{j_{p}}$ is an irreducible form of $t_{j_{1}} \ldots t_{k-1}$. 
Proof: All we must show is that $t_{j_{q}} t_{j_{q+1}} \nrightarrow 1$ for $1 \leq q \leq p-1$. Let $a^{(z)}=t_{j_{q}}$ and $b^{(u)}=t_{j_{q+1}}$. As $R$ links $a^{(z)}$ to $t_{p+q-1}$ the latter has the form $t_{p+q-1}=$ $a^{\prime(z+1)}$ for an appropriate $a^{\prime}$ in the connected component of $a$. Similarly, $t_{p+q}=$ $b^{\prime(u+1)}$ for some $b^{\prime}$ in the connected component of $b$. If $t_{j_{q}} t_{j_{q+1}} \rightarrow 1$, then $u=z+1$. Moreover, $a, a^{\prime}, b$ and $b^{\prime}$ belong to the same connected component and $a^{(z)}, a^{\prime(z+1)}, b^{(z+1)}$ and $b^{\prime(z+2)}$ occur in $T$. Then $a^{\prime(z+1)}$ cannot be a critical type because $T$ has complexity 2

Lemma 17. Assume that $R: t_{1} \ldots t_{n} \Rightarrow 1$, that $t_{k}$ is the leftmost critical type with corresponding link $\{j, k\}$ in $R$. Let $i$ be the top of the stack constructed by Lazy Parsing when processing $t_{k-1}$. Then $i \leq j \leq k-1$.

In particular, if $i<j$, then there are $l$ and $m$ such that $i<l<j, k<m$, Lazy Parsing links $t_{l}$ to $t_{j}, R$ links $t_{l}$ to $t_{m}$ and the latter is not a critical type.

Proof: Let $R: t_{1} \ldots t_{n} \Rightarrow 1, i, j$ and $k$ be as in the hypothesis of the Lemma. As $t_{k}$ is a critical type in a dictionary of complexity 2, it can only be a right endpoint, hence $j \leq k$. To see that $i \leq j$ assume that this was not the case, i.e. that

$$
\ldots t_{j} \ldots t_{i} \ldots t_{k} \ldots
$$

Hence $R$ links $i$ to some position $l$ strictly between $j$ and $k$. We remark first that $i<l$ is impossible. Indeed, Lazy Parsing reduces the segment $t_{i+1} \ldots t_{k-1}$ to the empty string by property (2) of Theorem (3). Hence it would link $l$ to some $p$ strictly between $i$ and $k$

$$
\ldots \underbrace{t_{i} \ldots \underbrace{t_{p} \ldots t_{l}}} \ldots t_{k} \ldots
$$

contradicting the uniqueness of links in the linear segment $t_{i} \ldots t_{k-1}$. Therefore $R$ must link $t_{i}$ to $t_{l}$ for some $l<i$.

$$
\ldots t_{j} \ldots t_{l} \ldots t_{i} \ldots t_{k} \ldots
$$

Next, we remark that $l$ is put on top of the stack while processing $t_{l}$, because $t_{l}$ does not contract with any type on its left. For if it did, $t_{i}$ would be a critical type, contradicting the assumption. When processing the string from $t_{l}$ toward the right, Lazy Parsing cannot pop $l$ from the stack before reaching $t_{i}$. Indeed, this would imply that $t_{l}$ is endpoint of two different links in a linear string, contradicting Lemma (7). It follows that Lazy Parsing computes an irreducible form of $t_{l} \ldots t_{i}$ different from the empty string, contradicting lemma (8). Therefore $j<i$ is impossible and so $i \leq j \leq k-1$.

Consider the case $i<j$. Recall Property (2) of Theorem (3) which says that Lazy Parsing reduces $t_{i+1} \ldots t_{k-1}$ to the empty string. Hence it links $j$ to some position $l$ with $i<l<k$. Moreover, we have $l<j$ because $t_{k}$ is the first critical type. Hence

$$
\ldots t_{i} \ldots \underbrace{t_{l} \ldots} t_{j} \ldots t_{k} \ldots
$$


where the underbraces designate links of Lazy Parsing. Note that $t_{l}$ is left-only, because the dictionary has complexity 2 . Therefore $R$ links $l$ to some $m>l$. Moreover, if we had $m \leq k-1$, then $l$ would be linked to two different positions in the linear string $t_{1} \ldots t_{k-1}$ which is impossible. Hence $k+1 \leq m$.

Lemma 18. Assume the notations of the preceding lemma and that $t_{k}$ is the leftmost critical type of a fan

$$
\ldots t_{j_{1}} \ldots \ldots t_{j_{p-1}} \ldots t_{j_{p}} \ldots t_{k} t_{k+1} \ldots t_{k+p-1} \ldots
$$

If $j_{p}>i$ then $j_{q}>i$ for $1 \leq q \leq p$. Moreover, there are $j_{1}>l_{1}>\cdots>l_{p}>i$ such that Lazy Parsing links $t_{l_{q}}$ to $t_{j_{q}}$.

Proof: Use induction on the number of critical types $p$. The case $p=1$ is the preceding lemma. For the induction step, omit the segment $t_{j_{p}} \ldots t_{k}$ from $t_{1} \ldots t_{n}$ and the corresponding links from $R$. Apply the induction hypothesis to this string to obtain $l_{p-1}<\cdots<l_{1}$ and the corresponding links of Lazy Parsing

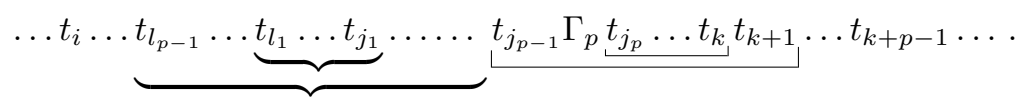

Use the usual argument to show that Lazy Parsing links $t_{l_{p}}$ to $t_{j_{p}}$ for some $l_{p}$ between $i$ and $j_{p}$

$$
\ldots t_{i} \ldots \underbrace{t_{l_{p}} \ldots} t_{j_{p} \ldots t_{k}} \ldots
$$

We have $l_{p}<l_{p-1}$ or $j_{p-1}<l_{p}$, for Lazy Parsing links $l_{p-1}$ to $j_{p-1}$. We remark that the latter is impossible. Indeed, if $t_{l_{p}}$ was inside of $\Gamma_{p}$ it would be linked by $R$ to some type before $t_{j_{p}}$ contradicting the uniqueness of links in the linear string $\Gamma_{p}$. Therefore $l_{p}<l_{p-1}$.

\section{Minimal Parsing}

Let $t_{1} \ldots t_{n}=T_{1} \ldots T_{m} s^{r}$ be a type assignment and assume that the dictionary is guarded. Then the first critical type is the leftmost type of a segment $C C^{\ell}$ within the type of some word. Hence the length of $C$ is a constant depending only on the dictionary.

We amend Lazy Parsing by back-tracking when arriving at the first critical type $t_{k}$ with $i$ on the top of the stack. Instead of processing $t_{k}$, we process the string $t_{i} \ldots t_{k-1}$ backward, starting at $k-1$, but no further than $i+1$ included. We construct a separate stack and set of links for the backward parsing. At each position, we compare the computed irreducible form, say $t_{j_{1}} \ldots t_{j_{q}}$, with the string $C^{\ell}$. If and when it satisfies $C^{\ell}=t_{j_{1}} \ldots t_{j_{p}}$, we stop and update Lazy Parsing following the construction of a minimal reduction in Theorem (15). We omit the links $\left\{l_{q}, j_{q}\right\}$ from the reduction computed so far and add the links $\left\{j_{q}, k+q-1\right\}$ and $\left\{l_{q}, k+p-1+q\right\}$ instead. The stack remains unchanged. Then we continue processing forward with Lazy Parsing from $t_{k+2 p}$ on. If on the contrary, the irreducible form computed by backtracking never becomes equal to $C^{\ell}$ for $k-1 \geq q \geq i+1$, we continue with forward Lazy Parsing from $t_{k}$ on, 
with the stack and set of links as they were before backtracking. If this procedure links the critical types successfully on the left, the corresponding segment $t_{j_{1}} \ldots t_{k+p-1}$ will be omitted in later backtracks. Hence every simple type in the string is processed at most twice.

It follows from Lemmas (17) and (18) that Minimal Parsing is complete if the dictionary is guarded and has complexity 2 . Moreover, it computes a reduction to the sentence time or fails if there is none, and does so in time proportional to the length of the string $T_{1} \ldots T_{m}$.

We can use Minimal Parsing for recognizing 'losing' type assignments with no reduction to the sentence type, when processing a word that introduces a critical type. If $k+q$ is pushed onto the stack for some $0 \leq q \leq p-1$, then $t_{1} \ldots t_{n}$ has no reduction to the empty string. Indeed, if Minimal Parsing does not find a link for the critical type $t_{k+q}$ then there is no such link by Theorem (15) and Lemmas (17) and (18). As $t_{k+q}$ cannot be cancelled from the right, it remains in every irreducible form of $t_{1} \ldots t_{n}$. Note that we do not even need to know what will come after the critical segment and its guard. We only need to know the critical segment $C$ so that we can test the irreducible form during backtracking for equality with $C^{\ell}$.

\section{Conclusion}

The conditions which make the Minimal Parsing algorithm linear apply to a large class of pregroup grammars. Indeed, a pregroup grammar has a finite dictionary and therefore is strongly equivalent to one of complexity 2. Practice shows that guarded dictionaries cover quite expressive natural language fragments. However, Minimal Parsing depends on the selected type assignment. Future work must investigate dictionaries for which the criterion for recognizing losing type assignments during processing lowers the number of processed type assignments sufficiently. The present work is only a first step toward the claim that pregroup grammars can provide natural language processing with linear algorithms.

\section{References}

[Buszkowski] Wojciech Buszkowski, Lambek Grammars based on pregroups, in: P. de Groote et al., editors, Logical Aspects of Computational Linguistics, LNAI 2099, Springer, 2001

[CYK] David Younger, Recognition and Parsing of Context-Free Languages in Time n3, Information and Control, 10:2, 1967

[Degeilh-Preller] Sylvain Degeilh, Anne Preller, Efficiency of Pregroups and the French noun phrase, Journal of Language, Logic and Information, Springer, Vol. 14, Number 4, pp. 423-444, 2005 
[Earley] Jay Earley, An efficient context-free parsing algorithm, Communications of the AMC, Volume 13, Number 2, pp 94-102, 1970

[Gazdar] Gerald Gazdar, Unbounded Dependency and Coordinate Structure, in: The Formal Complexity of Natural Language, Walter Salvitch, ed., Reidel Publishing Company, pp. 183-226, 1987

[Lambek 99] Joachim Lambek, Type Grammar revisited, in: A. Lecomte et al., editors, Logical Aspects of Computational Linguistics, Springer LNAI 1582, pp.1 -27, 1999

[Lambek 04] Joachim Lambek, A computational algebraic approach to English grammar, Syntax 7:2, pp. 128-147, 2004

[Oehrle] Richard Oehrle, A parsing algorithm for pregroup grammars, in: Proceedings of Categorial Grammars 2004, Montpellier France, pp.59-75, 2004

[Preller-Lambek] Anne Preller, Joachim Lambek (2007), Free compact 2categories, Mathematical Structures for Computer Sciences, vol. 17, pp.309-340, Cambridge University Press. doi:10.1017/S0960129506005901

[Preller 07] Anne Preller (2007), Toward Discourse Representation Via Pregroup Grammars, JoLLI, Vol.16, pp. 173-194. doi:10.1007/s10849-006-9033-y 